International Journal of Pure and Applied Mathematics

Volume 84 No. 3 2013, 175-183

ISSN: 1311-8080 (printed version); ISSN: 1314-3395 (on-line version)

url: http://www.ijpam.eu

doi: http://dx.doi.org/10.12732/ijpam.v84i3.4

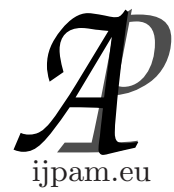

\title{
FRACTIONAL DIFFERENCE INEQUALITIES \\ OF GRONWALL-BELLMAN-BIHARI TYPE
}

\author{
J. Jagan Mohan ${ }^{1 \S}$, G.V.S.R. Deekshitulu² \\ ${ }^{1}$ Fluid Dynamics Division \\ School of Advanced Sciences \\ VIT University \\ Vellore, 632014, Tamil Nadu, INDIA \\ ${ }^{2}$ Department of Mathematics \\ JNTU Kakinada
}

Kakinada, 533003, Andhra Pradesh, INDIA

\begin{abstract}
The present paper obtains some new fractional order difference inequalities of the Gronwall-Bellman-Bihari type which can be used as a tool in applications.
\end{abstract}

AMS Subject Classification: 39A10, 39A99

Key Words: difference equations, fractional order, inequalities

\section{Introduction}

Fractional calculus has gained importance during the past three decades due to its applicability in diverse fields of science and engineering, such as, viscoelasticity, diffusion, neurology, control theory, and statistics. The analogous theory for discrete fractional calculus was initiated and properties of the theory of fractional sums and differences were established. Recently, a series of papers continuing this research has appeared. G.V.S.R. Deekshitulu and J. Jagan

Received: October 25, 2012

(c) 2013 Academic Publications, Ltd.

$\S_{\text {Correspondence author }}$ url: www.acadpubl.eu 
Mohan discussed some basic inequalities, comparison theorems and qualitative properties of the solutions of fractional difference equations [?, 3].

Throughout the present paper, for notations and terminology we use [1]. Now we introduce some basic definitions and results concerning nabla discrete fractional calculus.

Definition 1.1. The fractional sum operator of order $\alpha(\alpha \in \mathbb{R})$ is defined as

$$
\nabla^{-\alpha} u(n)=\sum_{j=0}^{n-1}\left(\begin{array}{c}
j+\alpha-1 \\
j
\end{array}\right) u(n-j)=\sum_{j=1}^{n}\left(\begin{array}{c}
n-j+\alpha-1 \\
n-j
\end{array}\right) u(j)
$$

and the fractional difference operator of order $\alpha(\alpha \in \mathbb{R}$ and $0<\alpha<1)$ is defined as

$$
\begin{aligned}
\nabla^{\alpha} u(n)=\sum_{j=0}^{n-1}\left(\begin{array}{c}
j-\alpha \\
j
\end{array}\right) & \nabla u(n-j) \\
= & \sum_{j=1}^{n}\left(\begin{array}{c}
n-j-\alpha-1 \\
n-j
\end{array}\right) u(j)-\left(\begin{array}{c}
n-\alpha-1 \\
n-1
\end{array}\right) u(0) .
\end{aligned}
$$

Clearly

$$
\nabla^{-\alpha} \nabla^{\alpha} u(n)=u(n)-u(0) \quad \text { and } \quad \nabla^{\alpha} \nabla^{-\alpha} u(n)=u(n) .
$$

Let $f(n, r): \mathbb{N}_{0}^{+} \times \mathbb{R} \rightarrow \mathbb{R}$. Consider a nonlinear difference equation of order $\alpha, 0<\alpha<1$, together with an initial condition is of the form $\nabla^{\alpha} u(n+1)=$ $f(n, u(n)), u(0)=u_{0}$. Now by replacing $u(n)$ by $\nabla^{\alpha} u(n)$ in the above equation, we have $u(n)=u_{0}+\sum_{j=0}^{n-1} B(n-1, \alpha ; j) f(j, u(j))$ where $B(n, \alpha ; j)=\left(\begin{array}{c}n-j+\alpha-1 \\ n-j\end{array}\right)$ for $0 \leq j \leq n$. Recently, the authors have established the following fractional order difference inequalities $[2,3,4]$.

Theorem 1.1. (Gronwall-Bellman Inequality) Let $u(n), a(n)$ and $b(n)$ be nonnegative real valued functions defined on $\mathbb{N}_{0}^{+}$. If, for $n \in \mathbb{N}_{0}^{+}$,

$$
\nabla^{\alpha} u(n+1) \leq a(n) u(n)+b(n)
$$

then

$$
\begin{aligned}
u(n) \leq u(0) & \prod_{j=0}^{n-1}[1+B(n-1, \alpha ; j) a(j)] \\
& \sum_{j=0}^{n-1} B(n-1, \alpha ; j) b(j) \prod_{k=j+1}^{n-1}[1+B(n-1, \alpha ; k) a(k)] .
\end{aligned}
$$


Definition 1.2. A function $H:[0, \infty) \rightarrow[0, \infty)$ is said to belong to the class $S$ if $H(u)$ is positive and nondecreasing for $u \geq 0$ and $\frac{1}{v} \leq H\left(\frac{u}{v}\right)$, for $u>0, v \geq 1$.

\section{Gronwall-Bellman-Bihari Type Inequalities}

The inequalities and comparison principles which provide explicit bounds on unknown functions play a very important role in the study of qualitative and quantitative properties of solutions of nonlinear difference equations. In this section, we shall establish some new fractional order difference inequalities of Gronwall-Bellman-Bihari type. Let $u(n), a(n), b(n), c(n)$ and $p(n)$ be nonnegative real valued functions defined on $\mathbb{N}_{0}^{+}$and $c \geq 0$ is a constant.

Lemma 2.1. If $a(n)$ is a positive, monotonic and nondecreasing function defined on $\mathbb{N}_{0}^{+}$and for $n \in \mathbb{N}_{0}^{+}$,

$$
u(n) \leq a(n)+\sum_{j=0}^{n-1} B(n-1, \alpha ; j) b(j)\left[u(j)+\sum_{k=0}^{j-1} B(j-1, \alpha ; k) c(k) u(k)\right]
$$

then

$$
\begin{aligned}
& u(n) \leq a(n)\left[1+\sum_{j=0}^{n-1} B(n-1, \alpha ; j) b(j)\right. \\
&\left.\prod_{k=0}^{j-1}[1+B(n-1, \alpha ; k)[b(k)+c(k)]]\right] .
\end{aligned}
$$

Proof. Since $a(n)$ is a positive, monotonic and nondecreasing function, from (2.1), we get

$$
\left.\frac{u(n)}{a(n)} \leq 1+\sum_{j=0}^{n-1} B(n-1, \alpha ; j) b(j)\left[\frac{u(j)}{a(j)}\right]+\sum_{k=0}^{j-1} B(j-1, \alpha ; k) c(k) \frac{u(k)}{a(k)}\right] .
$$

Define a function $z(n)$ by the right side of (2.3). Then $z(0)=1, \frac{u(n)}{a(n)} \leq z(n)$ and

$$
\nabla^{\alpha} z(n+1)=\leq b(n)\left[z(n)+\sum_{k=0}^{n-1} B(n-1, \alpha ; k) c(k) z(k)\right] .
$$


Let $v(n)=z(n)+\sum_{k=0}^{n-1} B(n-1, \alpha ; k) c(k) z(k)$. Then $v(0)=z(0)=1, z(n) \leq$ $v(n)$ and $\nabla^{\alpha} v(n+1)=\nabla^{\alpha} z(n+1)+c(n) z(n) \leq[b(n)+c(n)] v(n)$. Now an application of Theorem 1.1 yields

$$
v(n) \leq \prod_{j=0}^{n-1}[1+B(n-1, \alpha ; j)[b(j)+c(j)]]
$$

Then from (2.4) and (2.5), we have $\nabla^{\alpha} z(n+1) \leq b(n) \prod_{j=0}^{n-1}[1+B(n-$ $1, \alpha ; j)[b(j)+c(j)]]$. Now again by application of Theorem 1.1, we get

$$
z(n) \leq z(0)+\sum_{j=0}^{n-1} B(n-1, \alpha ; j)\left[b(j) \prod_{k=0}^{j-1}[1+B(j-1, \alpha ; k)[b(k)+c(k)]] .\right.
$$

Using (2.6) in $\frac{u(n)}{a(n)} \leq z(n)$, we get the required inequality in (2.2).

Theorem 2.2. Let $u(n)$ and $b(n)$ be positive real valued functions defined on $\mathbb{N}_{0}^{+}$and $a(n)$ is a positive, monotonic and nondecreasing function defined on $\mathbb{N}_{0}^{+}$and $H \in S$, for which the following inequality holds for $n \in \mathbb{N}_{0}^{+}$.

$$
u(n) \leq a(n)+\sum_{j=0}^{n-1} B(n-1, \alpha ; j) b(j)\left[u(j)+\sum_{k=0}^{j-1} B(j-1, \alpha ; k) b(k) H(u(k))\right],
$$

then

$$
\begin{aligned}
u(n) \leq a(n)\left[1+\sum_{j=0}^{n-1} B(n-1, \alpha ; j) b(j)\right. & \\
& \left.G^{-1}\left[G(1)+\sum_{k=0}^{j-1}[1+B(n-1, \alpha ; k) b(k)]\right]\right]
\end{aligned}
$$

for $0 \leq n \leq b$, where $\nabla^{\alpha} G(r(n+1))=\frac{\nabla^{\alpha} r(n+1)}{r(n)+H(r(n))}$ for $n \in \mathbb{N}_{0}^{+}$.

Here $r(n)$ is defined for $n \in \mathbb{N}_{0}^{+}, G^{-1}$ is the inverse of $G$ and $n$ is in the subinterval $[0, b]$ of $\mathbb{N}_{0}^{+}$so that $G(1)+\sum_{k=0}^{j-1}[1+B(n-1, \alpha ; k) b(k)] \in \operatorname{Dom}\left(G^{-1}\right)$.

Proof. Since $a(n)$ is a positive, monotonic and nondecreasing function, from (2.7), we get,

$$
\frac{u(n)}{a(n)} \leq 1+\sum_{j=0}^{n-1} B(n-1, \alpha ; j) b(j)\left[\frac{u(j)}{a(j)}+\sum_{k=0}^{j-1} B(j-1, \alpha ; k) b(k) H\left(\frac{u(k)}{a(k)}\right)\right] .
$$


Define a function $z(n)$ by the right side of (2.9). Then we have $z(0)=1$, $\frac{u(n)}{a(n)} \leq z(n)$ and $\nabla^{\alpha} z(n+1) \leq b(n)\left[z(n)+\sum_{k=0}^{n-1} B(n-1, \alpha ; k) b(k) H(z(k))\right]$. Let

$$
v(n)=z(n)+\sum_{k=0}^{n-1} B(n-1, \alpha ; k) b(k) H(z(k)) .
$$

Then $v(0)=z(0)=1, z(n) \leq v(n)$ and

$$
\nabla^{\alpha} v(n+1)=\nabla^{\alpha} z(n+1)+b(n) H(z(n)) \leq b(n)[v(n)+H(v(n))] .
$$

Dividing both sides of (2.11) by $v(n)+H(v(n))$ and using the hypothesis, we obtain $\nabla^{\alpha} G(v(n+1)) \leq b(n)$. Now an application of Theorem 1.1 yields

$$
G(v(n)) \leq G(1)+\sum_{j=0}^{n-1} B(n-1, \alpha ; j) b(j) .
$$

Then, we have $\nabla^{\alpha} z(n+1) \leq b(n) G^{-1}\left[G(1)+\sum_{j=0}^{n-1} B(n-1, \alpha ; j) b(j)\right]$. Now again by application of Theorem 1.1 , we get

$$
z(n) \leq 1+\sum_{j=0}^{n-1} B(n-1, \alpha ; j)\left[b(j) G^{-1}\left[G(1)+\sum_{k=0}^{j-1} B(j-1, \alpha ; k) b(k)\right]\right] .
$$

Using (2.13) in $\frac{u(n)}{a(n)} \leq z(n)$, we get the required inequality in (2.8). The interval $[0, b]$ is obvious.

Theorem 2.3. Let $W(u)$ be a positive, monotonic, nondecreasing and sub-multiplicative function for $u>0, W(0)=0$, and suppose further that the following inequality is satisfied for $n \in \mathbb{N}_{0}^{+}$, where $u(0)$ is a positive constant.

$$
\begin{aligned}
u(n) \leq u(0)+\sum_{j=0}^{n-1} B(n-1, \alpha ; j)[a(j) u(j) & +c(j) W(u(j)) \\
& \left.+a(j) \sum_{k=0}^{j-1} B(j-1, \alpha ; k) b(k) u(k)\right] .
\end{aligned}
$$

Then

$$
u(n) \leq A(n) G^{-1}\left[G(u(0))+\sum_{j=0}^{n-1} B(n-1, \alpha ; j) c(j) W(A(j))\right],
$$




$$
\text { for } 0 \leq n \leq b
$$

where

$$
A(n)=1+\sum_{j=0}^{n-1} B(n-1, \alpha ; j) a(j) \prod_{k=0}^{j-1}[1+B(n-1, \alpha ; k)[a(k)+b(k)]]
$$

and $\nabla^{\alpha} G(r(n+1))=\frac{\nabla^{\alpha} r(n+1)}{W(r(n))}$ for $n \in \mathbb{N}_{0}^{+}$.

Here $r(n)$ is defined for $n \in \mathbb{N}_{0}^{+}, G^{-1}$ is the inverse of $G$ and $n$ is in the subinterval $[0, b]$ of $\mathbb{N}_{0}^{+}$so that $G(u(0))+\sum_{j=0}^{n-1} B(n-1, \alpha ; j) c(j) W(A(j)) \in$ $\operatorname{Dom}\left(G^{-1}\right)$.

Proof. Define a function $z(n)$ by $z(n)=u(0)+\sum_{j=0}^{n-1} B(n-1, \alpha ; j) c(j) W(u(j))$. Then $z(0)=u(0), \nabla^{\alpha} z(n+1)=c(n) W(u(n))$ and

$$
\begin{aligned}
& u(n) \leq z(n) \\
& \quad+\sum_{j=0}^{n-1} B(n-1, \alpha ; j) a(j)\left[u(j)+\sum_{k=0}^{j-1} B(j-1, \alpha ; k) b(k) u(k)\right] .
\end{aligned}
$$

Since $z(n)$ is positive, monotonic, nondecreasing on $n \in \mathbb{N}_{0}^{+}$, from Lemma 2.1, we get

$$
\begin{array}{r}
u(n) \leq z(n)\left[1+\sum_{j=0}^{n-1} B(n-1, \alpha ; j) a(j) \prod_{k=0}^{j-1}[1+B(n-1, \alpha ; k)[a(k)+b(k)]]\right] \\
=z(n) A(n) .
\end{array}
$$

Since $W$ is sub-multiplicative, $W(u(n)) \leq W(z(n)) W(A(n))$. Hence $\frac{c(n) W(u(n))}{W(z(n))} \leq$ $c(n) W(A(n))$. Using the above facts in the hypothesis, we get $\nabla^{\alpha} G(z(n+1)) \leq$ $c(n) W(A(n))$. Now by application of Theorem 1.1, we get

$$
G(z(n)) \leq G(z(0))+\sum_{j=0}^{n-1} B(n-1, \alpha ; j) c(j) W(A(j)) .
$$

Substituting the value of $z(n)$ from (2.18) in (2.17), we get the required inequality in (2.14). The interval $[0, b]$ is obvious. 
Theorem 2.4. Let $H \in S$ and $W(u)$ be a positive, monotonic, nondecreasing and sub-multiplicative function for $u>0, W(0)=0$, and suppose that the following inequality is satisfied for $n \in \mathbb{N}_{0}^{+}$, where $u(0)$ is a positive constant.

$$
\begin{aligned}
u(n) \leq u(0)+\sum_{j=0}^{n-1} B(n-1, \alpha ; j)[a(j) u(j) & +b(j) W(u(j)) \\
& \left.+a(j) \sum_{k=0}^{j-1} B(j-1, \alpha ; k) a(k) H(u(k))\right] .
\end{aligned}
$$

Then

$$
\begin{aligned}
u(n) \leq B(n) \Omega^{-1}\left[\Omega(u(0))+\sum_{j=0}^{n-1} B(n-1, \alpha ; j) b(j) W(B(j))\right] & \\
& \text { for } 0 \leq n \leq b,
\end{aligned}
$$

where

$$
\begin{aligned}
& B(n)=1+\sum_{j=0}^{n-1} B(n-1, \alpha ; j) a(j) \\
& G^{-1}\left[G(1)+\sum_{k=0}^{j-1}[1+B(n-1, \alpha ; k) a(k)]\right]
\end{aligned}
$$

and $G, G^{-1}$ are defined as in Theorem 2.2, $\Omega$ is defined by $\nabla^{\alpha} \Omega(r(n+1))=$ $\frac{\nabla^{\alpha} r(n+1)}{W(r(n))}$ for $n \in \mathbb{N}_{0}^{+}$. Here $r(n)$ is defined for $n \in \mathbb{N}_{0}^{+}, \Omega^{-1}$ is the inverse of $\Omega$ and $n$ is in the subinterval $[0, b]$ of $\mathbb{N}_{0}^{+}$so that $G(1)+\sum_{l=0}^{k-1}[1+B(k-1, \alpha ; l) a(k)] \in$ $\operatorname{Dom}\left(G^{-1}\right)$ and $\Omega(u(0))+\sum_{j=0}^{n-1} B(n-1, \alpha ; j) b(j) W(B(j)) \in \operatorname{Dom}\left(\Omega^{-1}\right)$.

Proof. Define a function $z(n)$ by $z(n)=u(0)+\sum_{j=0}^{n-1} B(n-1, \alpha ; j) b(j) W(u(j))$. Then $z(0)=u(0), \nabla^{\alpha} z(n+1)=b(n) W(u(n))$ and

$$
\begin{aligned}
u(n) \leq z(n)+\sum_{j=0}^{n-1} B(n-1, \alpha ; j) a(j) & \\
& {\left[u(j)+\sum_{k=0}^{j-1} B(j-1, \alpha ; k) b(k) H(u(k))\right] . }
\end{aligned}
$$


Since $z(n)$ is positive, monotonic, nondecreasing on $n \in \mathbb{N}_{0}^{+}$, we have from Theorem 2.2,

$$
\begin{array}{r}
u(n) \leq z(n)\left[1+\sum_{j=0}^{n-1} B(n-1, \alpha ; j) a(j) G^{-1}\left[G(1)+\sum_{k=0}^{j-1}[1+B(n-1, \alpha ; k) a(k)]\right]\right] \\
=z(n) B(n) .
\end{array}
$$

Since $W$ is sub-multiplicative, $W(u(n)) \leq W(z(n)) W(B(n))$. Hence

$$
\frac{b(n) W(u(n))}{W(z(n))} \leq b(n) W(B(n)) .
$$

Using the above facts in the hypothesis, we get $\nabla^{\alpha} G(z(n+1)) \leq b(n) W(B(n))$. Now by application of Theorem 1.1, we get

$$
G(z(n)) \leq G(z(0))+\sum_{j=0}^{n-1} B(n-1, \alpha ; j) b(j) W(B(j)) .
$$

Substituting the value of $z(n)$ from (2.23) in (2.22), we get the required inequality in (2.19). The interval $[0, b]$ is obvious.

In the similar way one can prove the following theorem.

Theorem 2.5. Let $W(u)$ be a nondecreasing continuous and submultiplicative function defined on $\mathbb{R}$ with $W(u)>0$ for $u>0$. If for $n \in \mathbb{N}_{0}^{+}$,

$$
u(n) \leq c+p(n) \sum_{j=0}^{n-1} B(n-1, \alpha ; j) a(j) u(j)+\sum_{j=0}^{n-1} B(n-1, \alpha ; j) b(j) W(u(j))
$$

then

$$
\begin{aligned}
& u(n) \leq C(n) G^{-1}\left[G(c)+\sum_{j=0}^{n-1} B(n-1, \alpha ; j) b(j) W(C(j))\right] \\
& \qquad \text { for } 0 \leq n \leq b,
\end{aligned}
$$

where

$$
C(n)=1+p(n) \sum_{j=0}^{n-1} B(n-1, \alpha ; j) a(j)
$$




$$
\begin{aligned}
\left.\prod_{k=j+1}^{n-1}[1+B(n-1, \alpha ; k) a(k) p(k)]\right], \\
\nabla^{\alpha} G(r(n+1))=\frac{\nabla^{\alpha} r(n+1)}{W(r(n))} \text { for } n \in \mathbb{N}_{0}^{+} .
\end{aligned}
$$

Here $r(n)$ is defined for $n \in \mathbb{N}_{0}^{+}, G^{-1}$ is the inverse of $G$ and $n$ is in the subinterval $[0, b]$ of $\mathbb{N}_{0}^{+}$so that $G(c)+\sum_{j=0}^{n-1} B(n-1, \alpha ; j) b(j) g(A(j)) \in \operatorname{Dom}\left(G^{-1}\right)$.

\section{References}

[1] R.P. Agarwal, Difference Equations and Inequalities, Marcel Dekker, New York, 1992.

[2] G.V.S.R. Deekshitulu, J. Jagan Mohan, Fractional difference inequalities of Gronwall-Bellman type, Acta et Commentationes Universitatis Tartuensis de Mathematica, To Appear.

[3] G.V.S.R. Deekshitulu, J. Jagan Mohan, Some new fractional difference inequalities, ICMMSC 2012, CCIS, 283 (2012), Springer-Verlag, BerlinHeidelberg, 403-12.

[4] G.V.S.R. Deekshitulu, J. Jagan Mohan, Some new fractional difference inequalities of Gronwall-Bellman type, Mathematical Sciences, doi: 10.1186/2251-7456-6-69.

[5] V. Lakshmikantham, S. Leela, Differential and Integral Inequalities, Volume I, Academic Press, New York, 1969.

[6] B.G. Pachpatte, Inequalities of finite difference equations, Marcel Dekker, New York, 2002.

[7] I. Podlubny, Fractional Differential Equations, Academic press, San Diego, 1999. 
